2. Wiencek M. Beyond the naked eye: biofilms and microbial diversity in re-laundered mops and towels used in healthcare facilities. Am J Infect Control 2019;47:S4.

3. Sattar SA. Promises and pitfalls of recent advances in chemical means of preventing the spread of nosocomial infections by environmental surfaces. Am J Infect Control 2010;38:S34-S40.

4. Sattar SA, Maillard J-Y. The crucial role of wiping in decontamination of high-touch environmental surfaces: review of current status and directions for the future. Am J Infect Control 2013;41:S97-S104.
5. Sattar SA, Bradley C, Kibbee R, Wesgate R, Wilkinson MAC, Sharpe T, Maillard, J.-Y. Disinfectant wipes are appropriate to control microbial bioburden from surfaces- the use of a new ASTM standard test protocol to demonstrate efficacy. J Hosp Infect 2015;91:319-325.

6. Weber DJ, Anderson D, Rutala WA. The role of the surface environment in healthcare-associated infections. Curr Opin Infect Dis 2013;26:338-344.

\title{
Improving antibiotic use through antimicrobial stewardship interventions upon discharge
}

\author{
Cynthia T. Nguyen PharmD ${ }^{1}$, Angela M. Huang PharmD ${ }^{2}$ and Jerod L. Nagel PharmD \\ ${ }^{1}$ Department of Pharmacy, University of Chicago Medicine, Chicago, Illinois, ${ }^{2}$ Department of Pharmacy, HonorHealth John C. Lincoln Medical Center, Phoenix, \\ Arizona and ${ }^{3}$ Department of Pharmacy, Michigan Medicine, Ann Arbor, Michigan
}

To the Editor-Employing antimicrobial stewardship principles at every phase of patient care is crucial. Although much of the antimicrobial stewardship literature is focused among inpatients, Dyer et $\mathrm{al}^{1}$ have identified an important opportunity to measure and reduce antimicrobial exposure postdischarge. Excessive outpatient antibiotic therapy for treatment of pneumonia is associated with increased risk for adverse effects. ${ }^{2}$ As such, we wanted to share additional outcomes related to our multicenter evaluation of an antimicrobial stewardship initiative focused on duration of therapy (DOT) for $>600$ patients with community-acquired pneumonia (CAP), ${ }^{3}$ which is consistent with the findings by Dyer et $\mathrm{al}^{3}$ and highlights the widespread need to focus on stewardship practices across phases of care.

This initiative employed a multifaceted intervention including institutional guideline update, provider education using educational sessions and pocket cards, and prospective audit with feedback and intervention. Prospective audit with feedback and intervention was performed by infectious diseases pharmacists Monday through Friday. Interventions were made to recommend durations of therapy consistent with the 2007 IDSA and American Thoracic Society (IDSA/ATS) CAP guidelines, including a focus on postdischarge prescriptions. ${ }^{4}$ Following this intervention, we observed a reduction in the median total DOT (6 vs 9 days; $P<.001)$. Importantly, this change was attributed to a significant reduction in postdischarge DOT ( 3 vs 5 days; $P<.001$ ). The inpatient DOT ( 3 vs 3 days; $P=.217$ ) and hospital length of stay ( 3 vs 4 days; $P=.060$ ) remained similar before and after the inter- vention. Consequently, the percentage of postdischarge days accounting for overall antimicrobial exposure for CAP was reduced from $64 \%$ to $50 \%(P<.001)$. Our findings support the call for antimicrobial stewardships programs to target antimicrobial prescribing at transitions of care and demonstrate that interventions upon discharge can reduce overall antimicrobial exposure.

\section{Acknowledgments.}

Financial support. No financial support was provided relevant to this article.

Conflicts of interest. All authors report no conflicts of interest relevant to this article.

\section{References}

1. Dyer AP, Dodds Ashley E, Anderson DJ, et al. Total duration of antimicrobial therapy resulting from inpatient hospitalization. Infect Control Hosp Epidemiol 2019;40:847-854.

2. Vaughn VM, Flanders SA, Snyder A, et al. Excess antibiotic treatment duration and adverse events in patients hospitalized with pneumonia: a multihospital cohort study. Ann Intern Med 2019;171:153-163.

3. Foolad F, Huang AM, Nguyen CT, et al. A multicentre stewardship initiative to decrease excessive duration of antibiotic therapy for the treatment of community-acquired pneumonia. J Antimicrob Chemother 2018;73:1402-1407.

4. Mandell LA, Wunderink RG, Anzueto A, et al. Infectious Diseases Society of America/American Thoracic Society consensus guidelines on the management of community-acquired pneumonia in adults. Clin Infect Dis 2007;44 suppl 2:S27-S72.

\footnotetext{
Address for correspondence: Cynthia T. Nguyen, PharmD, Department of Pharmacy, University of Chicago Medicine, 5841 S Maryland Ave, MC0010 TE019, Chicago, IL 60637-1470. Email: CynthNguyen@gmail.com

Cite this article: Nguyen CT, Huang AM, and Nagel JL. (2019). Improving antibiotic use through antimicrobial stewardship interventions upon discharge. Infection Control \& Hospital Epidemiology, 40: 1327, https://doi.org/10.1017/ice.2019.246
} 